\title{
EXPLICIT BOUNDS FOR SUMS OF SQUARES
}

\author{
JEREMY ROUSE
}

\begin{abstract}
For an even integer $k$, let $r_{2 k}(n)$ be the number of representations of $n$ as a sum of $2 k$ squares. The quantity $r_{2 k}(n)$ is approximated by the classical singular series $\rho_{2 k}(n) \asymp n^{k-1}$. Deligne's bound on the Fourier coefficients of Hecke eigenforms gives that $r_{2 k}(n)=\rho_{2 k}(n)+O\left(d(n) n^{\frac{k-1}{2}}\right)$. We determine the optimal implied constant in this estimate provided that either $k / 2$ or $n$ is odd. The proof requires a delicate positivity argument involving Petersson inner products.
\end{abstract}

\section{Introduction and statement of results}

In Hardy's book on Ramanujan [1], he states the following (Chapter 9, p. 132).

The problem of the representations of an integer $n$ as the sum of a given number $k$ of integral squares is one of the most celebrated in the theory of numbers. Its history may be traced back to Diophantus, but begins effectively with Girard's (or Fermat's) theorem that a prime $4 m+1$ is the sum of two squares. Almost every arithmetician of note since Fermat has contributed to the solution of the problem, and it has its puzzles for us still.

If $n$ is a non-negative integer, let

$$
r_{s}(n)=\#\left\{\left(x_{1}, x_{2}, \ldots, x_{s}\right) \in \mathbb{Z}^{s}: x_{1}^{2}+x_{2}^{2}+\cdots+x_{s}^{2}=n\right\}
$$

be the number of representations of $n$ as a sum of $s$ squares.

The classical work that Hardy refers to includes the results of Jacobi giving the following exact formulae. Let $n$ be a positive integer and write $n=2^{\alpha} m$, where $m$ is odd. Then

$$
r_{4}(n)=\left\{\begin{array}{ll}
8 \sigma_{1}(m), & \text { if } \alpha=0, \\
24 \sigma_{1}(m), & \text { if } \alpha \geq 1,
\end{array} \quad r_{8}(n)= \begin{cases}16 \sigma_{3}(m), & \text { if } \alpha=0 \\
16 \cdot \frac{2^{3 \alpha+3}-15}{7} \sigma_{3}(m), & \text { if } \alpha \geq 1\end{cases}\right.
$$

The search for higher exact formulae (each involving more complicated arithmetic functions) for was carried out by many mathematicians. Glaisher [2] and Rankin [3] were interested in these formulae where the arithmetic functions involved were multiplicative.

In a different direction, Hardy [4] and Mordell [5] applied the circle method to give an approximation

$$
r_{s}(n)=\rho_{s}(n)+R_{s}(n)
$$

where $\rho_{s}(n)$ is the "singular series" and $R_{s}(n)$ is an error term. Here $\rho_{s}(n)$ can be expressed as a divisor sum if $s$ is even, and $\rho_{s}(n) \asymp n^{\frac{s}{2}-1}$ provided $s>4$. The

Received by the editors August 8, 2011.

2010 Mathematics Subject Classification. Primary 11E25; Secondary 11F30. 
contribution $R_{s}(n)$ is more mysterious, and Deligne's proof of the Weil conjectures (see [6]) implies an estimate of the form

$$
R_{s}(n)=O\left(d(n) n^{\frac{s}{4}-\frac{1}{2}}\right)
$$

provided $s$ is even. The phenomena of exact formulae for $r_{s}(n)$ of the form $r_{s}(n)=$ $\rho_{s}(n)$ only occurs for small $s$. In [7], Rankin shows that $R_{s}(n)$ is identically zero if and only if $s \leq 8$. Exact formulae of a different nature were given by Milne in [8] when $s=4 n^{2}$ and $s=4 n(n+1)$.

The problem we study is the implied constant in equation (1.1) above. This is a natural question, and in [9-11], the author has studied the corresponding problem for powers of the $\Delta$ function, $p$-core partitions (joint work with Byungchan Kim), and arbitrary level 1 cusp forms (joint work with Paul Jenkins), respectively. To prove their now famous "290-theorem" Bhargava and Hanke [12] compute this implied constant for about 6000 quadratic forms in four variables and use this to determine precisely which integers these forms represent.

Returning to our problem, if $s=2 k$ and $k$ is even, we have that

$$
\begin{aligned}
& \rho_{2 k}(n) \\
& \quad=\frac{2 k(-1)^{k / 2+1}}{\left(2^{k}-1\right) B_{k}}\left(\sigma_{k-1}(n)+\left(-1+(-1)^{k / 2+1}\right) \sigma_{k-1}(n / 2)+(-1)^{k / 2} 2^{k} \sigma_{k-1}(n / 4)\right),
\end{aligned}
$$

where $B_{k}$ is the $k$ th Bernoulli number and $\sigma_{k-1}(n)$ is the sum of the $(k-1)$ st powers of the positive integer divisors of $n$ (and is hence zero if $n$ is not an integer). Our main result is the following.

Theorem 1.1. Suppose that $k$ is even. If either $k / 2$ is odd or $n$ is odd, then we have

$$
\left|r_{2 k}(n)-\rho_{2 k}(n)\right| \leq\left(4 k+\frac{2 k(-1)^{k / 2}}{\left(2^{k}-1\right) B_{k}}\right) d(n) n^{\frac{k-1}{2}}
$$

Remark 1.1. If $2 k=4$ or $2 k=8$, the right hand side is zero, and we recover the exact formulae of Jacobi. For arbitrary even $k$, we have $r_{2 k}(1)=4 k$ and $\rho_{2 k}(1)=$ $\frac{2 k(-1)^{k / 2+1}}{\left(2^{k}-1\right) B_{k}}$. Thus, the inequality above becomes an equality when $n=1$. This shows that the implied constant

$$
4 k+\frac{2 k(-1)^{k / 2}}{\left(2^{k}-1\right) B_{k}}
$$

in (1.1) is best possible. The error term is smaller than the main term provided $n \gg k^{2}$.

Our approach to proving Theorem 1.1 is as follows. If

$$
\theta(z)=1+2 \sum_{n=1}^{\infty} q^{n^{2}}, \quad q=\mathrm{e}^{2 \pi \mathrm{i} z}
$$

is the classical Jacobi theta function, then

$$
\theta^{2 k}(z)=\sum_{n=0}^{\infty} r_{2 k}(n) q^{n}
$$


is a modular form of weight $k$ on $\Gamma_{0}(4)$. If $k$ is even, we can decompose

$$
\theta^{2 k}(z)=a_{1} E_{k}(z)+a_{2} E_{k}(2 z)+a_{3} E_{k}(4 z)+\sum_{i} c_{i} g_{i}(z)+\sum_{i} d_{i} g_{i}(2 z)+\sum_{i} e_{i} g_{i}(4 z)
$$

where

$$
E_{k}(z)=1-\frac{2 k}{B_{k}} \sum_{n=1}^{\infty} \sigma_{k-1}(n) q^{n}
$$

is the classical level 1 Eisenstein series, and the $g_{i}(z)$ are normalized newforms of level 1,2 or 4 . We prove the following.

Theorem 1.2. Assume the notation above. Then for all $i, c_{i} \geq 0$.

Theorem 1.2 allows us to read off

$$
\sum_{i}\left|c_{i}\right|=\sum_{i} c_{i}=4 k+\frac{2 k(-1)^{k / 2}}{\left(2^{k}-1\right) B_{k}}
$$

from the coefficient of $q$ on both sides of (1.2), using that $a_{1}=\frac{(-1)^{k / 2}}{\left(2^{k}-1\right) B_{k}}$.

To prove Theorem 1.2 we use properties of the Petersson inner product on $M_{k}\left(\Gamma_{0}(4)\right)$ (see Section 2 for precise definitions). If $g_{i}(z)$ is a newform of level 4 , then $g_{i}(z)$ is orthogonal to every other term in the expansion (1.2). It follows that

$$
\left\langle\theta^{2 k}, g_{i}\right\rangle=c_{i}\left\langle g_{i}, g_{i}\right\rangle \text {. }
$$

It suffices to prove that $\left\langle\theta^{2 k}, g_{i}\right\rangle \geq 0$. This Petersson inner product consists of a contribution from each of the three cusps of $\Gamma_{0}(4): \infty, 0$ and $1 / 2$. The contribution from $\infty$ is

$$
\frac{2}{(4 \pi)^{k}} \sum_{n=1}^{\infty} \frac{r_{2 k}(n) a(n)}{n^{k-1}} \int_{4 \pi n}^{\infty} u^{k-2} \mathrm{e}^{-u} d u .
$$

Here $g_{i}(z)=\sum_{n=1}^{\infty} a(n) q^{n}$. Our approach is to show that the main term in the above sum comes from $n=1$. If $n$ is fixed, $r_{2 k}(n)$ is a polynomial of degree $2 k$ in $n$. We compute these polynomials explicitly, and use this to the bound the terms when $2 \leq n \leq 2500$. Next, we use a simple induction bound on $r_{2 k}(n)$ to show that the terms with $2500 \leq n \leq \frac{k}{2 \pi} \log (k)$ are small enough. Finally, we use the exponential decay of $\int_{4 \pi n}^{\infty} u^{k-2} \mathrm{e}^{-u} d u$ when $n \geq \frac{k}{2 \pi} \log (k)$.

The cusp at zero behaves in an essentially identical way to the cusp at $\infty$, and the contribution from the cusp at $1 / 2$ is very small, since $\theta(z)$ vanishes there.

Remark 1.2. This result can be thought of as a refined form of the circle method. The Eisenstein series is the contribution of the major arcs, while Deligne's result, and the bounds we give on the constants $c_{i}$ can be thought of as explicit, uniform minor arc estimates. Further, it is plausible that the Fourier coefficients of distinct newforms are independent (an assertion that could be justified under the assumption of the holomorphy of certain Rankin-Selberg convolutions). This combined with the recent proof of the Sato-Tate conjecture (see [13]) suggests that for any $\epsilon>0$, there are infinitely many primes $p$ so that

$$
\left|r_{2 k}(p)-\rho_{2 k}(p)\right|>\left(4 k+\frac{2 k(-1)^{k / 2}}{\left(2^{k}-1\right) B_{k}}-\epsilon\right) d(p) p^{\frac{k-1}{2}} .
$$


Remark 1.3. The proof gives more detailed information about the constants $c_{i}$ in (1.2). In particular, if $g_{i}(z)$ is a newform of level 4 and $k \equiv 2(\bmod 4)$, then

$$
c_{i}=16 k \cdot \frac{(k-2) !}{(4 \pi)^{k}\left\langle g_{i}, g_{i}\right\rangle}\left(1+O\left(\alpha^{k}\right)\right),
$$

where $\alpha \approx 0.918$. If $k \equiv 0(\bmod 4)$, then $c_{i}=0$. Similar, but more complicated results are true for the constants $c_{i}$ associated with levels 1 and 2 newforms.

An outline of the paper is as follows. In Section 2, we give precise definitions and review necessary background information. In Section 3, we prove a number of auxiliary results that will be used in the proof of Theorem 1.2. In Section 4, we prove Theorem 1.2 and use this to deduce Theorem 1.1. Finally in Section 5, we address other values of $k$ and $n$.

\section{Background}

In this section, we give definitions and review necessary background. For $N \geq 1$, let $M_{k}\left(\Gamma_{0}(N)\right)$ denote the $\mathbb{C}$-vector space of modular forms of weight $k$ on $\Gamma_{0}(N):=$ $\left\{\left(\begin{array}{ll}a & b \\ c & d\end{array}\right) \in \mathrm{SL}_{2}(\mathbb{Z}): N \mid c\right\}$. Let $S_{k}\left(\Gamma_{0}(N)\right)$ denote the subspace of cusp forms.

If $f$ is a modular form of weight $k$, and $\alpha=\left[\begin{array}{ll}a & b \\ c & d\end{array}\right] \in \mathrm{GL}_{2}(\mathbb{Q})$ and has positive determinant, define the usual slash operator by

$$
f \mid \alpha=(a d-b c)^{k / 2}(c z+d)^{-k} f\left(\frac{a z+b}{c z+d}\right) .
$$

For a positive integer $d$, define the operator $V(d)$ by $f(z) \mid V(d)=f(d z)$. It is wellknown (see [14], p. 107 for a proof) that $V(d)$ maps $M_{k}\left(\Gamma_{0}(N)\right)$ to $M_{k}\left(\Gamma_{0}(d N)\right)$ and $S_{k}\left(\Gamma_{0}(N)\right)$ to $S_{k}\left(\Gamma_{0}(d N)\right)$. For a positive integer $d$, define the operator $U(d)$ by

$$
\sum_{n=0}^{\infty} a(n) q^{n} \mid U(d)=\sum_{n=0}^{\infty} a(d n) q^{n}
$$

If $d \mid N$, then $U(d)$ maps $M_{k}\left(\Gamma_{0}(N)\right)$ to itself and $S_{k}\left(\Gamma_{0}(N)\right)$ to itself. If $p$ is a prime with $p \nmid N$, define the usual Hecke operator $T(p)$ by $T(p)=U(p)+p^{k-1} V(p)$.

If $f, g \in M_{k}\left(\Gamma_{0}(N)\right)$ and at least one of $f$ or $g$ is a cusp form, let

$$
\langle f, g\rangle=\frac{3}{\pi\left[\mathrm{SL}_{2}(\mathbb{Z}): \Gamma_{0}(N)\right]} \iint_{\mathbb{H} / \Gamma_{0}(N)} f(x+\mathrm{i} y) \overline{g(x+\mathrm{i} y)} y^{k} \frac{d x d y}{y^{2}}
$$

denote the usual Petersson inner product. If $p \nmid N$, then the Hecke operators $T(p)$, acting on $S_{k}\left(\Gamma_{0}(N)\right)$, are self-adjoint with respect to the Petersson inner product. Moreover, if $\alpha \in \mathrm{GL}_{2}(\mathbb{Q})$ and has positive determinant, then $\langle f|\alpha, g| \alpha\rangle=\langle f, g\rangle$.

Let $S_{k}^{\text {new }}\left(\Gamma_{0}(N)\right)$ denote the orthogonal complement under this inner product of the space spanned by all forms

$$
f(z) \mid V(d), \text { where } f(z) \in S_{k}\left(\Gamma_{0}(M)\right),
$$

and we have $M \mid N, M<N$, and $d$ is a divisor of $N / M$. A newform of level $N$ is a form

$$
f(z)=\sum_{n=1}^{\infty} a(n) q^{n} \in S_{k}^{\text {new }}\left(\Gamma_{0}(N)\right)
$$


that is a simultaneous eigenform of the Hecke operators $T(p)$, normalized so that $a(1)=1$. We have the Deligne bound

$$
|a(n)| \leq d(n) n^{\frac{k-1}{2}}
$$

where $d(n)$ is the number of divisors of $n$ (for a detailed proof of this inequality, see the new book by Brian Conrad [15]). A newform $f(z)$ of level $N$ is also an eigenform of the Atkin-Lehner operator $W_{N}=\left[\begin{array}{cc}0 & -1 \\ N & 0\end{array}\right]$. This operator commutes with the Hecke operators $T(p)$ for primes $p \nmid N$. One has more information about the coefficient $a(p)$ if $p \mid N$. If $N=p$, then $a(p)=-\lambda p^{\frac{k}{2}-1}$, where $\lambda$ is the eigenvalue of $f$ under $W_{N}$. If $p^{2} \mid N$, then $a(p)=0$ (see [16], Theorem 3).

The multiplicity-one theorem states that the joint eigenspaces of all $T(p)$ (with $p \nmid N)$ in $S_{k}^{\text {new }}\left(\Gamma_{0}(N)\right)$ are one-dimensional. It follows from this, and the self-adjointness of the Hecke operators, that if $f_{1}$ and $f_{2}$ are two distinct newforms, then $\left\langle f_{1}, f_{2}\right\rangle=0$. It is known (see Section 5.11 of [17]) that the Eisenstein series $E_{k}(z)$ (and $E_{k}(z) \mid V(d)$ ) are orthogonal to cusp forms under the Petersson inner product.

Finally, let $\eta(z)$ denote as usual the Dedekind eta function

$$
\eta(z)=q^{1 / 24} \prod_{n=1}^{\infty}\left(1-q^{n}\right), \quad q=\mathrm{e}^{2 \pi \mathrm{i} z} .
$$

We have the following well-known identities:

$$
\begin{aligned}
\theta(z) & =\frac{\eta^{5}(2 z)}{\eta^{2}(z) \eta^{2}(4 z)}, \\
\frac{\eta^{8}(4 z)}{\eta^{4}(2 z)} & =\sum_{n=0}^{\infty} \sigma(2 n+1) q^{2 n+1}, \\
(2 z+1)^{-2} \theta^{4}\left(\frac{z}{2 z+1}\right) & =16 \frac{\eta^{8}(4 z)}{\eta^{4}(2 z)}
\end{aligned}
$$

(see the exercises on page 145 of [18], solutions are on p. 234).

\section{Preliminary results}

In this section, we prove three lemmas that will be used in the proof of the main results. Our first lemma proves some simple bounds on $r_{s}(n)$.

Lemma 3.1. (1) Suppose that $n$ is a non-negative integer. There are non-negative constants $c_{i, n}(0 \leq i \leq n)$ so that

$$
r_{s}(n)=\sum_{i=0}^{n} c_{i, n}\left(\begin{array}{l}
s \\
i
\end{array}\right), \text { for all } s \geq 0 .
$$

(2) If $n$ is fixed, $\frac{r_{2 s}(n)}{n^{\frac{s-1}{2}}}$ is a decreasing function of $s$, provided $2 s \geq n+\frac{n}{\sqrt[4]{n}-1}$.

(3) If $n$ is a positive integer and $s \geq 6$, then

$$
r_{s}(n) \leq \frac{3(4.11)^{s}}{25 \sqrt{s !}}(n+s)^{\frac{s}{2}-1} \text {. }
$$


Proof. We prove the first statement by strong induction on $n$. For $n=0$, we have $r_{s}(0)=1=1 \cdot\left(\begin{array}{l}s \\ 0\end{array}\right)$. Thus, $c_{0,0}=1$ and the result holds.

Assume the result is true for all $m<n$. Let $t$ be a positive integer with $t \leq s$. Then

$$
\begin{aligned}
r_{t}(n)-r_{t-1}(n) & =2 \sum_{r=1}^{\lfloor\sqrt{n}\rfloor} r_{t-1}\left(n-r^{2}\right) \\
& =2 \sum_{r=1}^{\lfloor\sqrt{n}\rfloor} \sum_{i=0}^{n-r^{2}} c_{i, n-r^{2}}\left(\begin{array}{c}
t-1 \\
i
\end{array}\right) .
\end{aligned}
$$

Summing both sides over all $t, 1 \leq t \leq s$ and using that $\sum_{t=1}^{s}\left(\begin{array}{c}t-1 \\ i\end{array}\right)=\left(\begin{array}{c}s \\ i+1\end{array}\right)$ gives

$$
\begin{aligned}
r_{s}(n) & =\sum_{r=1}^{\lfloor\sqrt{n}\rfloor} \sum_{i=0}^{n-r^{2}} 2 c_{i, n-r^{2}}\left(\begin{array}{c}
s \\
i+1
\end{array}\right) \\
& =2 \sum_{i=1}^{n}\left(\sum_{r=1}^{\lfloor\sqrt{n-i}\rfloor} c_{i-1, n-r^{2}}\right)\left(\begin{array}{l}
s \\
i
\end{array}\right) .
\end{aligned}
$$

Since the $c_{i-1, n-r^{2}}$ are non-negative, by the induction hypothesis, it follows that their sum is non-negative, and this proves that the result is true for $n$.

To prove the second statement, it suffices to prove that each term in the expression

$$
\frac{r_{2 s}(n)}{n^{\frac{s-1}{2}}}=\sum_{i=0}^{n} c_{i, n} \frac{\left(\begin{array}{c}
2 s \\
i
\end{array}\right)}{n^{\frac{s-1}{2}}}
$$

is a decreasing function of $s$. Let $f(s)=\left(\begin{array}{c}2 s \\ i\end{array}\right) \cdot n^{(1-s) / 2}$. Then,

$$
\begin{aligned}
\frac{f(s+1)}{f(s)} & =\frac{1}{\sqrt{n}} \cdot \frac{(2 s+2)(2 s+1)}{(2 s+2-i)(2 s+1-i)} \\
& \leq \frac{1}{\sqrt{n}} \frac{(2 s+2)(2 s+1)}{(2 s+2-n)(2 s+1-n)} \\
& <\frac{1}{\sqrt{n}}\left(1+\frac{n}{2 s-n}\right)^{2} .
\end{aligned}
$$

This is a decreasing function of $s$, and if we take $s=n+\frac{n}{\sqrt[4]{n}-1}$, then $2 s-n=\frac{n}{\sqrt[4]{n}-1}$ and so

$$
\frac{1}{\sqrt{n}}\left(1+\frac{n}{2 s-n}\right)^{2}=\frac{1}{\sqrt{n}}(1+(\sqrt[4]{n}-1))^{2}=1
$$

This proves that $f(s+1)<f(s)$, as desired.

We prove the third statement by induction on $s$. Our base case is $s=6$ and in this case, we use the exact formula:

$$
r_{6}(n)=\sum_{d \mid n} d^{2}\left(-4 \chi_{-1}(d)+16 \chi_{-1}(n / d)\right),
$$


where

$$
\chi_{-1}(n)= \begin{cases}1, & \text { if } n \equiv 1 \quad(\bmod 4) \\ -1, & \text { if } n \equiv 3 \quad(\bmod 4) \\ 0, & \text { if } n \text { is even }\end{cases}
$$

We rewrite this as

$$
r_{6}(n)=n^{2} \sum_{d \mid n} \frac{16 \chi_{-1}(n / d)-4 \chi_{-1}(d)}{(n / d)^{2}} .
$$

If $n$ is even, then $r_{6}(n) / n^{2} \leq 8 \zeta(2) \leq 13.2$. On the other hand if $n$ is odd, then $16 \chi_{-1}(n / d)-4 \chi_{-1}(d)$ is negative if $n / d \equiv 3(\bmod 4)$ and $16 \chi_{-1}(n / d)-4 \chi_{-1}(d) \leq 20$ if $n / d \equiv 1(\bmod 4)$. Thus,

$$
\frac{r_{6}(n)}{n^{2}} \leq 20 \sum_{\substack{d \mid n \\(\bmod 4)}} \frac{1}{d^{2}} \leq 20 \sum_{n=0}^{\infty} \frac{1}{(4 n+1)^{2}} .
$$

One can show that the right-hand side above is about $21.4966613 \leq \frac{6449}{300}$. We denote by $C_{s}$ a constant so that $r_{s}(n) \leq C_{s}(n+s)^{\frac{s}{2}-1}$, and we take $C_{6}=\frac{6449}{300}$. This proves the base case.

Assume now that $s \geq 6$. We have

$$
\begin{aligned}
r_{s+1}(n) & =r_{s}(n)+2 \sum_{m=1}^{\lfloor\sqrt{n}\rfloor} r_{s}\left(n-m^{2}\right) \\
& \leq C_{s}(n+s)^{\frac{s}{2}-1}+2 C_{s} \sum_{m=1}^{\lfloor\sqrt{n}\rfloor}\left(n+s-m^{2}\right)^{\frac{s}{2}-1} \\
& \leq C_{s}(n+s)^{\frac{s}{2}-1}+2 C_{s} \int_{0}^{\sqrt{n+s+1}}\left(n+s+1-x^{2}\right)^{\frac{s}{2}-1} d x \\
& \leq C_{s}(n+s)^{\frac{s}{2}-1}+2 C_{s}(n+s+1)^{\frac{s+1}{2}-1} \int_{0}^{1}\left(1-u^{2}\right)^{\frac{s}{2}-1} d u .
\end{aligned}
$$

We have

$$
\left(1-u^{2}\right)^{\frac{s}{2}-1}=\mathrm{e}^{\left(\frac{s}{2}-1\right) \log \left(1-u^{2}\right)} \leq \mathrm{e}^{-(s / 2-1) u^{2}} .
$$

Thus

$$
2 \int_{0}^{1}\left(1-u^{2}\right)^{\frac{s}{2}-1} d u \leq 2 \int_{0}^{\infty} \mathrm{e}^{-(s / 2-1) u^{2}} d u=\sqrt{\frac{\pi}{\frac{s}{2}-1}}
$$

and

$$
\begin{aligned}
r_{s+1}(n) & \leq C_{s}(n+s)^{\frac{s}{2}-1}+C_{s}(n+s+1)^{\frac{s+1}{2}-1}\left[\sqrt{\frac{2 \pi}{s-2}}\right] \\
& \leq C_{s}(n+s+1)^{\frac{s+1}{2}-1}\left[\sqrt{\frac{2 \pi}{s-2}}+\frac{(n+s)^{(s / 2)-1}}{(n+s+1)^{\frac{s+1}{2}-1}}\right] .
\end{aligned}
$$


Note that the second term inside the brackets above is a decreasing function of $n$ and is relevant only for $n \geq 1$. It follows that

$$
\begin{aligned}
r_{s+1}(n) & \leq C_{s}(n+s+1)^{\frac{s+1}{2}-1} \cdot \frac{1}{\sqrt{s+1}}\left[\sqrt{2 \pi} \sqrt{\frac{s+1}{s-2}}+\left(\frac{s+1}{s+2}\right)^{s / 2-1}\right] \\
& \leq \frac{C_{s} \cdot 4.11}{\sqrt{s+1}}(n+s+1)^{\frac{s+1}{2}-1} .
\end{aligned}
$$

Hence, we may take $C_{s+1}=\frac{4.11}{\sqrt{s+1}} C_{s}$ and so

$$
C_{s}=\frac{6449}{300} \cdot \frac{4.11^{s-6}}{\sqrt{s ! / 6 !}} \leq \frac{3(4.11)^{s}}{25 \sqrt{s !}} .
$$

Next, we use Deligne's bound on the Fourier coefficients of a newform to bound its value.

Lemma 3.2. Suppose that $k \geq 7, y \geq \frac{1}{2 \pi}$, and $g(z)=\sum_{n=1}^{\infty} a(n) q^{n}$ with $|a(n)| \leq$ $d(n) n^{\frac{k-1}{2}}$. Then

$$
|g(x+\mathrm{i} y)| \leq \frac{1}{(2 \pi y)^{\frac{k+1}{2}}} \Gamma\left(\frac{k+1}{2}\right)\left[\log \left(\frac{k+1}{2}\right)+\gamma+1\right],
$$

where $\gamma$ is Euler's constant.

Proof. Since the $n$th Fourier coefficient of $g(z)$ is bounded by $d(n) n^{\frac{k-1}{2}}$, we have that

$$
|g(x+\mathrm{i} y)| \leq \sum_{n=1}^{\infty} d(n) n^{\frac{k-1}{2}} \mathrm{e}^{-2 \pi n y}
$$

If $D(x)=\sum_{n \leq x} d(n)$, then $D(x) \leq x \log (x)+\gamma x+1 \leq x \log (x)+(\gamma+1) x$. By partial summation, we have

$$
\begin{aligned}
\sum_{n=1}^{\infty} d(n) n^{\frac{k-1}{2}} \mathrm{e}^{-2 \pi n y} & =\int_{1}^{\infty} D(x)\left[2 \pi y x^{\frac{k-1}{2}}-\left(\frac{k-1}{2}\right) x^{\frac{k-3}{2}}\right] \mathrm{e}^{-2 \pi x y} d x \\
& \leq 2 \pi y \int_{\frac{k-1}{4 \pi y}}^{\infty}(\log (x)+(\gamma+1)) x^{\frac{k+1}{2}} \mathrm{e}^{-2 \pi x y} d x .
\end{aligned}
$$

Now, we set $u=2 \pi x y, d u=2 \pi y d x$. We get

$$
\begin{aligned}
& 2 \pi y \int_{\frac{k-1}{2}}^{\infty}\left(\log \left(\frac{u}{2 \pi y}\right)+(\gamma+1)\right)\left(\frac{u}{2 \pi y}\right)^{\frac{k+1}{2}} \mathrm{e}^{-u} \frac{d u}{2 \pi y} \\
& =\frac{1}{(2 \pi y)^{\frac{k+1}{2}}} \int_{\frac{k-1}{2}}^{\infty}(\log (u)-\log (2 \pi y)+\gamma+1) u^{\frac{k+1}{2}} \mathrm{e}^{-u} d u
\end{aligned}
$$

Since $y \geq \frac{1}{2 \pi}, \log (2 \pi y)>0$ and so we neglect the term involving it. We get

$$
\frac{1}{(2 \pi y)^{\frac{k+1}{2}}}\left[\int_{\frac{k-1}{2}}^{\infty} \log (u) u^{\frac{k+1}{2}} \mathrm{e}^{-u} d u+(\gamma+1) \int_{\frac{k-1}{2}}^{\infty} u^{\frac{k+1}{2}} \mathrm{e}^{-u} d u\right]
$$


If we extend the integrals down to zero, then the negative contribution of $\int_{0}^{1} \log (u)$ $u^{\frac{k+1}{2}} \mathrm{e}^{-u} d u$ is cancelled by that of $[1.5,2]$ for $k \geq 7$. Thus, we get the bound

$$
|g(z)| \leq \frac{1}{(2 \pi y)^{\frac{k+1}{2}}} \Gamma\left(\frac{k+1}{2}\right)\left[\psi\left(\frac{k+1}{2}\right)+\gamma+1\right],
$$

where $\Gamma^{\prime}(z)=\int_{0}^{\infty} \log (u) u^{z-1} \mathrm{e}^{-u} d u$ and $\psi(z)=\frac{\Gamma^{\prime}(z)}{\Gamma(z)}$. The formula (see equation 6.3.21 on p. 258 of [19])

$$
\psi(z)=\log (z)-\frac{1}{2 z}-\int_{0}^{\infty} \frac{2 t d t}{\left(z^{2}+t^{2}\right)\left(\mathrm{e}^{2 \pi t}-1\right)}
$$

shows that $\psi(z) \leq \log (z)$. Thus, we obtain the bound

$$
\frac{1}{(2 \pi y)^{\frac{k+1}{2}}} \Gamma\left(\frac{k+1}{2}\right)\left[\log \left(\frac{k+1}{2}\right)+\gamma+1\right] \text {. }
$$

Finally, we will need to understand Petersson inner products of newforms $f$ with their images under $V(d)$. This is the subject of the next result.

Lemma 3.3. Suppose that $f(z)=\sum_{n=1}^{\infty} a(n) q^{n} \in S_{k}^{\text {new }}\left(\Gamma_{0}(N)\right)$ is a newform. If $p \nmid N$, then

$$
\langle f, f \mid V(p)\rangle=\frac{a(p)}{p^{k-1}(p+1)}\langle f, f\rangle .
$$

Note that the assumption that $f$ has trivial character implies that the Fourier coefficients of $f$ are real. This fact will be used frequently in what follows.

Proof. Rankin proved in [20] that if $f=\sum a(n) q^{n}$ and $g=\sum b(n) q^{n}$ are cusp forms of weight $k$, then

$$
\sum_{n \leq x} \frac{a(n) \overline{b(n)}}{n^{k-1}}=\frac{(4 \pi)^{k}}{(k-1) !}\langle f, g\rangle x+O\left(x^{3 / 5}\right) .
$$

We will use this formula to prove the results above. We start by letting $c=\frac{(4 \pi)^{k}}{(k-1) !}$, and $p$ be a prime number with $p \nmid N$. Then,

$$
\begin{aligned}
\langle f, f \mid V(p)\rangle & =\lim _{x \rightarrow \infty} \frac{1}{c} \cdot \frac{1}{x} \sum_{n \leq x} \frac{a(n) a(n / p)}{n^{k-1}} \\
& =\lim _{x \rightarrow \infty} \frac{1}{c} \cdot \frac{1}{x} \sum_{p n \leq x} \frac{a(p n) a(n)}{(p n)^{k-1}} \\
& =\lim _{x \rightarrow \infty} \frac{1}{c} \cdot \frac{1}{p^{k}} \cdot \frac{1}{\frac{x}{p}} \sum_{n \leq \frac{x}{p}} \frac{a(p n) a(n)}{n^{k-1}} \\
& =\frac{1}{p^{k}}\langle f, f \mid U(p)\rangle .
\end{aligned}
$$


Now, $a(p) f=f|T(p)=f| U(p)+p^{k-1} f \mid V(p)$. It follows that

$$
\begin{aligned}
a(p)\langle f, f\rangle & =\langle f, f \mid T(p)\rangle=\langle f, f \mid U(p)\rangle+p^{k-1}\langle f, f \mid V(p)\rangle \\
& =p^{k}\langle f, f \mid V(p)\rangle+p^{k-1}\langle f, f \mid V(p)\rangle \\
& =p^{k-1}(p+1)\langle f, f \mid V(p)\rangle .
\end{aligned}
$$

Thus,

$$
\langle f, f \mid V(p)\rangle=\frac{a(p)}{p^{k-1}(p+1)}\langle f, f\rangle .
$$

\section{Proof of Theorems 1.1 and $\mathbf{1 . 2}$}

In this section, we will prove the main results. We will first prove Theorem 1.2 and then deduce Theorem 1.1 from it.

Proof of Theorem 1.2. First, for each newform $g$ of level 1, 2 or 4 , we will find a form $\tilde{g}$ with the property that the coefficient of $g$ in the representation of $\theta^{2 k}$ is positive if and only if $\left\langle\theta^{2 k}, \tilde{g}\right\rangle>0$. Each $\tilde{g}$ will be an eigenform of $T_{p}$ for all odd primes $p$, and will also be an eigenform of $W_{4}$ with the same eigenvalue as that of $\theta^{2 k}$.

Recall the decomposition

$$
\theta^{2 k}(z)=a_{1} E_{k}(z)+a_{2} E_{k}(2 z)+a_{3} E_{k}(4 z)+\sum_{i} c_{i} g_{i}(z)+\sum_{i} d_{i} g_{i}(2 z)+\sum_{i} e_{i} g_{i}(4 z),
$$

where the $g_{i}$ are newforms of levels 1,2 or 4 , and the $c_{i}, d_{i}, e_{i} \in \mathbb{R}$. If $V$ is an eigenspace for all $T_{n}$ (with $n$ odd), then $V$ is also stable under $W_{4}$. Since $\theta^{2 k} \mid W_{4}=(-1)^{\frac{k}{2}} \theta^{2 k}$, it follows that the projection of $\theta^{2 k}$ onto $V$ must also have eigenvalue $(-1)^{\frac{k}{2}}$ under $W_{4}$.

If $V$ is an eigenspace coming from a newform $g_{i}$ of level 4 , then $\operatorname{dim} V=1$. If $c_{i} \neq 0$, then $g_{i} \mid W_{4}=(-1)^{\frac{k}{2}}$. In this case, we have $\left\langle\theta^{2 k}, g\right\rangle=\left\langle c_{i} g_{i}, g_{i}\right\rangle=c_{i}\left\langle g_{i}, g_{i}\right\rangle$ and thus $c_{i}>0$ if and only if $\left\langle\theta^{2 k}, g_{i}\right\rangle>0$, and so we set $\tilde{g}_{i}=g_{i}$. Part (i) of Theorem 7 of [16] shows that for any newform of level $4, g_{i} \mid W_{4}=-g_{i}$, and hence $c_{i}=0$, if $k \equiv 0$ $(\bmod 4)$.

If $V$ is an eigenspace coming from a newform $g_{i}$ of level 2 , then $\operatorname{dim} V=2$. This vector space decomposes into one-dimensional plus and minus eigenspaces under the action of $W_{4}$. It follows that the projection of $\theta^{2 k}$ onto $V$ is $c_{i}\left(g_{i}+(-2)^{\frac{k}{2}} \lambda g_{i} \mid V(2)\right)$, where $\lambda$ is the Atkin-Lehner eigenvalue of $g_{i}$. Thus, we set $\tilde{g}_{i}=g_{i}+(-2)^{\frac{k}{2}} \lambda g_{i} \mid V(2)$. This form will be orthogonal to any element in the opposite $W_{4}$ eigenspace, since $W_{4}$ is an isometry with respect to the Petersson inner product. It follows that $c_{i}>0$ if and only if $\left\langle\theta^{2 k}, \tilde{g}_{i}\right\rangle>0$.

If $V$ is an eigenspace coming from a newform $g_{i}$ of level 1 , then $\operatorname{dim} V=3$ and

$$
\begin{aligned}
g_{i} \mid W_{4} & =2^{k} g_{i} \mid V(4), \\
g_{i}|V(2)| W_{4} & =g_{i} \mid V(2), \\
g_{i}|V(4)| W_{4} & =2^{-k} g_{i} .
\end{aligned}
$$

We have that $V=V^{+} \oplus V^{-}$, where $V^{+}$and $V^{-}$are the plus and minus eigenspaces for $W_{4}$. Then $\operatorname{dim} V^{+}=2$ and it is spanned by $g_{i}+2^{k} g_{i} \mid V(4)$ and $g_{i} \mid V(2)$. Also $\operatorname{dim} V^{-}=1$ and it is spanned by $g_{i}-2^{k} g_{i} \mid V(4)$. If $k \equiv 0(\bmod 4)$, then the AtkinLehner sign is +1 . If $k \equiv 2(\bmod 4)$, the Atkin-Lehner sign is -1 . 
When $k \equiv 2(\bmod 4)$, we set $\tilde{g}_{i}=g_{i}-2^{k} g_{i} \mid V(4)$. This form satisfies $\tilde{g}_{i} \mid W_{4}=-\tilde{g}_{i}$, and is again orthogonal to the form spanning the plus eigenspace for $W_{4}$.

When $k \equiv 0(\bmod 4)$, we set $\tilde{g}_{i}=g_{i}-\frac{4}{3} a(2) g_{i}\left|V(2)+2^{k} g_{i}\right| V(4)$. This form satisfies $\tilde{g}_{i} \mid W_{4}=\tilde{g}_{i}$, and is hence orthogonal to $g_{i}-2^{k} g_{i} \mid V(4)$. By Lemma 3.3 it is orthogonal to $g_{i} \mid V(2)$.

We have

$$
\begin{aligned}
\left\langle\theta^{2 k}, \tilde{g}_{i}\right\rangle & =\frac{1}{2 \pi} \iint_{\mathbb{H} / \Gamma_{0}(4)} \theta^{2 k}(z) \overline{\tilde{g}_{i}(z)} y^{k} \frac{d x d y}{y^{2}} \\
& =\frac{1}{2 \pi} \sum_{j=1}^{6} \int_{-1 / 2}^{1 / 2} \int_{\sqrt{1-x^{2}}}^{\infty}\left(\left.\theta^{2 k}\right|_{k} \gamma_{j}\right)(x+\mathrm{i} y) \overline{\left.\tilde{g}_{i}\right|_{k} \gamma_{j}(x+\mathrm{i} y)} y^{k-2} d y d x
\end{aligned}
$$

Here, the matrices

$$
\begin{array}{ll}
\gamma_{1}=\left[\begin{array}{ll}
1 & 0 \\
0 & 1
\end{array}\right], & \gamma_{2}=\left[\begin{array}{cc}
0 & -1 \\
1 & 0
\end{array}\right], \quad \gamma_{3}=\left[\begin{array}{cc}
0 & -1 \\
1 & 1
\end{array}\right], \\
\gamma_{4}=\left[\begin{array}{cc}
0 & -1 \\
1 & 2
\end{array}\right], & \gamma_{5}=\left[\begin{array}{cc}
0 & -1 \\
1 & 3
\end{array}\right], \quad \gamma_{6}=\left[\begin{array}{ll}
1 & 0 \\
2 & 1
\end{array}\right]
\end{array}
$$

are a set of representatives for the right cosets of $\Gamma_{0}(4)$ in $\mathrm{SL}_{2}(\mathbb{Z})$.

Term 1. This is the contribution from the cusp at infinity. In particular, it is the $j=1$ term in the above sum. We split this term into two parts: $\{x+\mathrm{i} y:-1 / 2 \leq x \leq$ $1 / 2, y \geq 1\}$, and $\left\{x+\mathrm{i} y:-1 / 2 \leq x \leq 1 / 2, \sqrt{1-x^{2}} \leq y \leq 1\right\}$.

Write

$$
\tilde{g}_{i}(z)=\sum_{n=1}^{\infty} a(n) q^{n}
$$

Applying the Deligne bound to each of the various possible forms of $\tilde{g}_{i}$, we see that in all cases $|a(n)| \leq \frac{17}{3} d(n) n^{\frac{k-1}{2}}$.

The first part is

$$
\frac{1}{2 \pi} \int_{1}^{\infty} \int_{-1 / 2}^{1 / 2}\left(\sum_{m=0}^{\infty} r_{2 k}(m) \mathrm{e}^{-2 \pi m y} \mathrm{e}^{2 \pi i m x}\right)\left(\sum_{n=1}^{\infty} \overline{a(n)} \mathrm{e}^{-2 \pi n y} \mathrm{e}^{-2 \pi \mathrm{i} n x}\right) y^{k-2} d x d y .
$$

Since the Fourier series representations converge uniformly on compact subsets of these regions, we can invert the summations and the integrals and obtain

$$
\frac{1}{2 \pi} \sum_{m=0}^{\infty} \sum_{n=1}^{\infty} r_{2 k}(m) \overline{a(n)} \int_{-1 / 2}^{1 / 2} \int_{1}^{\infty} y^{k-2} \mathrm{e}^{-2 \pi(m+n) y} \mathrm{e}^{2 \pi i(m-n) x} d y d x .
$$

The integral over $-1 / 2 \leq x \leq 1 / 2$ is zero unless $m=n$, in which case it is 1 . We set $u=4 \pi n y, d u=4 \pi n d y$ and this gives

$$
\frac{2}{(4 \pi)^{k}} \sum_{n=1}^{\infty} \frac{r_{2 k}(n) \overline{a(n)}}{n^{k-1}} \int_{4 \pi n}^{\infty} u^{k-2} \mathrm{e}^{-u} d u
$$


We now split this sum into several ranges. The main contribution comes from $n=1$. We have $a(1)=1$ and $r_{2 k}(1)=4 k$. This term is

$$
\begin{aligned}
\frac{8 k}{(4 \pi)^{k}} \int_{4 \pi}^{\infty} u^{k-2} \mathrm{e}^{-u} d u & =\frac{8 k}{(4 \pi)^{k}}\left[\int_{0}^{\infty} u^{k-2} \mathrm{e}^{-u} d u-\int_{0}^{4 \pi} u^{k-2} \mathrm{e}^{-u} d u\right] \\
& \geq \frac{8 k}{(4 \pi)^{k}}\left[(k-2) !-(4 \pi)^{k-1} \mathrm{e}^{-4 \pi}\right],
\end{aligned}
$$

for $k \geq 15$, since if $k>4 \pi+2, u^{k-2} \mathrm{e}^{-u}$ is increasing on [0, $\left.4 \pi\right]$.

The second range is $2 \leq n \leq 2500$. Here we explicitly compute the polynomials $r_{2 k}(n)$ (using the algorithm in the proof of part 1 of Lemma 3.1). Part 2 of Lemma 3.1 shows that $\frac{r_{2 k}(n)}{n^{\frac{k-1}{2}}}$ is a decreasing function of $k$, provided $k \geq 1456$.

The third range is $2500 \leq n \leq \frac{k}{2 \pi} \log (2 k)$. In this range, we use the bound from part 3 of Lemma 3.1, the Deligne bound, $d(n) \leq 2 \sqrt{n}$, and we obtain that

$$
\begin{aligned}
\left|\frac{r_{2 k}(n) \overline{a(n)}}{n^{k-1}}\right| & \leq \frac{34(4.11)^{2 k}}{25 \sqrt{(2 k) !}} \cdot \sqrt{n} \cdot \frac{(n+2 k)^{k-1}}{n^{\frac{k-1}{2}}} \\
& \leq \frac{34}{25} \frac{(4.11)^{2 k}}{\sqrt{(2 k) !}} \cdot \sqrt{\frac{k}{2 \pi} \log (2 k)} \cdot\left(\sqrt{n}+\frac{2 k}{\sqrt{n}}\right)^{k-1} .
\end{aligned}
$$

The function $f(x)=\left(x+\frac{2 k}{x}\right)^{k-1}$ is decreasing for $x<\sqrt{2 k}$ and increasing after that. We have that $f(50)=f\left(\frac{2 k}{50}\right)$ and $\frac{2 k}{50} \geq \sqrt{\frac{k}{2 \pi} \log (2 k)}$ if $k \geq 724$. Thus, we have the bound

$$
\frac{68}{25} \frac{(4.11)^{2 k}}{(4 \pi)^{k} \sqrt{(2 k) !}} \cdot \frac{k^{3 / 2}}{(2 \pi)^{3 / 2}} \log ^{3 / 2}(2 k) \cdot\left(50+\frac{2 k}{50}\right)^{k-1} \cdot(k-2) !
$$

valid provided $k \geq 724$. For $k \leq 724$, we use the larger of the values of $f$ at $x=50$ and $x=\sqrt{\frac{k}{2 \pi} \log (2 k)}$.

The fourth and final range is $n \geq \frac{k}{2 \pi} \log (2 k)$. In this range we use the decay of the integral $\int_{4 \pi n}^{\infty} u^{k-2} \mathrm{e}^{-u} d u$. We have that $u \geq 2 k \log (2 k)$ and so $u^{k-2} \mathrm{e}^{-u} \leq \mathrm{e}^{-u / 2}$ and so the integral is bounded by $2 \mathrm{e}^{-2 \pi n}$. Bounding $\overline{a(n)}$ and $r_{2 k}(n)$ as before, we have that the contribution from this range is at most

$$
\frac{34}{3(4 \pi)^{k}} \sum_{n=\frac{k}{2 \pi} \log (2 k)}^{\infty} \frac{2 n^{\frac{k}{2}}}{n^{k-1}} \cdot\left(\frac{3}{25} \cdot \frac{(4.11)^{2 k}}{\sqrt{(2 k) !}}\right)(n+2 k)^{k-1} \cdot 2 \mathrm{e}^{-2 \pi n} .
$$

We write $\frac{(n+2 k)^{k-1}}{n^{k-1}}$ as $\left(1+\frac{2 k}{n}\right)^{k-1}$. If $k \geq 40,1+\frac{2 k}{n} \leq 3.87$ and we get

$$
\frac{136}{25(4 \pi)^{k}} \cdot \frac{(4.11)^{2 k}(3.87)^{k-1}}{\sqrt{(2 k) !}} \sum_{n=\frac{k}{2 \pi} \log (2 k)}^{\infty} n^{\frac{k}{2}} \mathrm{e}^{-2 \pi n}
$$

If $a_{n}=n^{\frac{k}{2}} \mathrm{e}^{-2 \pi n}$, then we have

$$
\frac{a_{n+1}}{a_{n}} \leq\left(1+\frac{1}{n}\right)^{\frac{k}{2}} \mathrm{e}^{-2 \pi} \leq \mathrm{e}^{\frac{k}{2 n}-2 \pi} \leq \mathrm{e}^{-2 \pi+\frac{\pi}{\log (2 k)}} \leq \mathrm{e}^{-5.6} .
$$


Thus, we get the bound

$$
\frac{136}{25(4 \pi)^{k}} \cdot \frac{(4.11)^{2 k}(3.87)^{k-1}}{\sqrt{(2 k) !}} \cdot\left(\frac{k}{2 \pi} \log (2 k)\right)^{\frac{k}{2}}(2 k)^{-k} \cdot \frac{1}{1-\mathrm{e}^{-5.6}},
$$

valid if $k \geq 40$.

The second part of the contribution from the cusp at infinity is

$$
\frac{1}{2 \pi} \int_{-1 / 2}^{1 / 2} \int_{\sqrt{1-x^{2}}}^{1} \theta^{2 k}(x+\mathrm{i} y) \overline{g(x+\mathrm{i} y)} y^{k-2} d y d x
$$

In this region we use Lemma 3.2 to bound $g(x+\mathrm{i} y)$, and we use that

$$
|\theta(z)| \leq 1+2 \sum_{n=1}^{\infty} \mathrm{e}^{-2 \pi n^{2} y} \leq 1.008667
$$

for $y \geq \sqrt{3} / 2$. This gives the bound

$$
\frac{\Gamma\left(\frac{k+1}{2}\right)\left[\log \left(\frac{k+1}{2}\right)+\gamma+1\right](1.008667)^{2 k}}{(2 \pi)^{\frac{k+3}{2}}} \int_{-1 / 2}^{1 / 2} \int_{\sqrt{1-x^{2}}}^{1} y^{\frac{k-5}{2}} d y d x .
$$

The double integral above is less than or equal to $\int_{-1 / 2}^{1 / 2} \int_{0}^{1} y^{\frac{k-5}{2}} d y d x=\frac{2}{k-3}$. Hence, we get the bound

$$
\frac{34 \Gamma\left(\frac{k+1}{2}\right)\left[\log \left(\frac{k+1}{2}\right)+\gamma+1\right](1.008667)^{2 k}}{3(k-3)(2 \pi)^{\frac{k+3}{2}}},
$$

valid for $k \geq 7$.

Term 2. This is the contribution of the cusp at zero, and in particular the contributions from the terms involving $\gamma_{2}, \gamma_{3}, \gamma_{4}$, and $\gamma_{5}$. We have

$$
\theta^{2 k} \mid W_{4}=(-1)^{\frac{k}{2}} \theta^{2 k} \quad \text { and } \quad \tilde{g}_{i} \mid W_{4}=(-1)^{\frac{k}{2}} \tilde{g}_{i}
$$

Translating this into Fourier expansions gives

$$
\theta^{2 k}\left|\left[\begin{array}{cc}
0 & -1 \\
1 & 0
\end{array}\right]=\frac{(-1)^{\frac{k}{2}}}{2^{k}} \theta^{2 k}\left(\frac{z}{4}\right), \quad \tilde{g}_{i}\right|\left[\begin{array}{cc}
0 & -1 \\
1 & 0
\end{array}\right]=\frac{(-1)^{\frac{k}{2}}}{2^{k}} \tilde{g}_{i}\left(\frac{z}{4}\right) .
$$

Thus, the contribution from these four terms is

$$
\frac{1}{(2 \pi) \cdot 4^{k}} \sum_{j=0}^{3} \int_{-1 / 2}^{1 / 2} \int_{\sqrt{1-x^{2}}}^{\infty} \theta^{2 k}\left(\frac{x+j+\mathrm{i} y}{4}\right) \overline{\tilde{g}_{i}\left(\frac{x+j+\mathrm{i} y}{4}\right)} y^{k-2} d y d x .
$$

We set $u=x / 4$ and $v=y / 4$ in the integrand and obtain

$$
\frac{1}{2 \pi} \sum_{j=0}^{3} \int_{-1 / 8}^{1 / 8} \int_{\frac{\sqrt{1-16 u^{2}}}{4}}^{\infty} \theta^{2 k}\left(u+\mathrm{i} v+\frac{j}{4}\right) \overline{\tilde{g}_{i}\left(u+\mathrm{i} v+\frac{j}{4}\right)} v^{k-2} d v d u .
$$

We break this into two terms. The first term consists of those pieces with $v \leq 1$. The smallest value $v$ takes on this piece is $\sqrt{3} / 8$ and since $\sqrt{3} / 8>\frac{1}{2 \pi}$, we may use Lemma 3.2 to bound the contribution. This yields

$$
\left|\tilde{g}_{i}(u+i v)\right| \leq \frac{17}{3 \cdot(2 \pi v)^{\frac{k+1}{2}}} \Gamma\left(\frac{k+1}{2}\right)\left[\log \left(\frac{k+1}{2}\right)+\gamma+1\right] .
$$


We also have

$$
|\theta(u+\mathrm{i} v)| \leq 1+2 \sum_{n=1}^{\infty} \mathrm{e}^{-2 \pi n^{2} v} \leq 1.52182
$$

for $v \geq \sqrt{3} / 8$. The contribution of these terms is therefore bounded by

$$
\frac{17 \cdot(1.52182)^{2 k}}{3 \cdot(2 \pi)^{\frac{k+3}{2}}} \Gamma\left(\frac{k+1}{2}\right)\left[\log \left(\frac{k+1}{2}\right)+\gamma+1\right] \sum_{j=0}^{3} \int_{-1 / 8}^{1 / 8} \int_{\frac{\sqrt{1-16 u^{2}}}{4}}^{1} v^{\frac{k-5}{2}} d v d u
$$

The sum of double integrals is bounded by $\int_{-1 / 2}^{1 / 2} \int_{0}^{1} v^{\frac{k-5}{2}} d v=\frac{2}{k-3}$ and we get the bound

$$
\frac{34 \cdot(1.52182)^{2 k}}{3 \cdot(2 \pi)^{\frac{k+3}{2}} \cdot(k-3)} \Gamma\left(\frac{k+1}{2}\right)\left[\log \left(\frac{k+1}{2}\right)+\gamma+1\right]
$$

on the part where $v \leq 1$, valid for $k \geq 7$.

The second term consists of those pieces with $v \geq 1$. This gives

$$
\frac{1}{2 \pi} \int_{-1 / 2}^{1 / 2} \int_{1}^{\infty} \theta^{2 k}(u+\mathrm{i} v) \overline{\tilde{g}_{i}(u+\mathrm{i} v)} v^{k-2} d v d u
$$

This is exactly the same as the contribution of the first part of the cusp at infinity!

Term 3. This is the contribution of the cusp at $1 / 2$ corresponding to the matrix $\gamma_{6}$. We must understand the Fourier expansion of $\tilde{g}_{i} \mid \gamma_{6}$. Since $\gamma_{6} \in \Gamma_{0}(2)$, terms of level 1 or 2 are not affected.

If $g$ is a newform of level 4 , then since $\gamma_{6}$ is not in $\Gamma_{0}(4)$, we have that $g \mapsto g+g \mid \gamma_{6}$ is the trace map from $S_{k}\left(\Gamma_{0}(4)\right)$ to $S_{k}\left(\Gamma_{0}(2)\right)$. Since newforms are in the kernel of the trace map (by Theorem 4 of [21]), it follows that $g+g \mid \gamma_{6}=0$ and so $g \mid \gamma_{6}=-g$.

If $g$ is a newform of level 2, we have

$$
g|V(2)| \gamma_{6}=2^{-k / 2} g\left|\left[\begin{array}{ll}
2 & 0 \\
0 & 1
\end{array}\right]\left[\begin{array}{ll}
1 & 0 \\
2 & 1
\end{array}\right]=2^{-k / 2} g\right|\left[\begin{array}{cc}
0 & -1 \\
2 & 0
\end{array}\right]\left[\begin{array}{cc}
1 & 0 \\
-2 & 1
\end{array}\right]\left[\begin{array}{cc}
1 & 1 / 2 \\
0 & 1
\end{array}\right] .
$$

The first matrix is the Atkin-Lehner involution of level 2, of which $g$ is an eigenform. The second matrix is in $\Gamma_{0}(2)$ and the third matrix does not affect the size of the Fourier coefficients at infinity. It follows that the $n$th Fourier coefficient of $g|V(2)| \gamma_{6}$ is bounded by $2^{-k / 2} d(n) n^{\frac{k-1}{2}}$.

If $g$ is a newform of level 1 , we have

$$
g|V(4)| \gamma_{6}=2^{-k} g\left[\begin{array}{ll}
4 & 0 \\
0 & 1
\end{array}\right]\left[\begin{array}{cc}
1 & 0 \\
2 & 1
\end{array}\right]=2^{-k} g \mid\left[\begin{array}{cc}
2 & 1 \\
1 & 1
\end{array}\right]\left[\begin{array}{cc}
2 & -1 \\
0 & 2
\end{array}\right]=2^{-k} g(z-1 / 2) .
$$

Thus, the $n$th Fourier coefficient of $g|V(4)| \gamma_{6}$ is bounded by $2^{-k} d(n) n^{\frac{k-1}{2}}$. It follows that for any $\tilde{g}_{i}$, the $n$th coefficient of $\tilde{g}_{i} \mid \gamma_{6}$ is bounded by $\frac{14}{3} d(n) n^{\frac{k-1}{2}}$.

Now, $\theta^{2 k} \mid \gamma_{6}=2^{2 k} \frac{\eta(4 z)^{4 k}}{\eta(2 z)^{4 k}}$. The form $F(z)=\frac{\eta(4 z)^{8}}{\eta(2 z)^{4}} \in M_{2}\left(\Gamma_{0}(4)\right)$ and satisfies

$$
F(z)=\sum_{n \text { odd }} \sigma(n) q^{n}
$$

Thus, for $y \geq \sqrt{3} / 2,|F(z)| \leq \mathrm{e}^{-2 \pi y}\left(\sum_{n \text { odd }} \sigma(n) \mathrm{e}^{-2 \pi(n-1) y}\right) \leq 1.0001 \mathrm{e}^{-2 \pi y}$ and so

$$
\left|\theta^{2 k}\right| \gamma_{6} \mid \leq 2^{2 k}(1.0001)^{k / 2} \mathrm{e}^{-k \pi y}
$$


for $y \geq \sqrt{3} / 2$. The contribution of the cusp at $1 / 2$ is therefore

$$
\frac{1}{2 \pi} \int_{-1 / 2}^{1 / 2} \int_{\sqrt{1-x^{2}}}^{\infty} \theta^{2 k} \mid \gamma_{6}(x+\mathrm{i} y) \overline{\tilde{g}_{i} \mid \gamma_{6}(x+\mathrm{i} y)} y^{k-2} d y d x .
$$

By Lemma 3.2, we have

$$
\left|\tilde{g}_{i}\right| \gamma_{6}(x+\mathrm{i} y) \mid \leq \frac{14}{3} \frac{1}{(2 \pi)^{\frac{k+1}{2}}} \Gamma\left(\frac{k+1}{2}\right)\left[\log \left(\frac{k+1}{2}\right)+\gamma+1\right] \cdot \frac{1}{y^{\frac{k+1}{2}}} .
$$

This gives the bound

$$
\frac{14 \cdot 2^{2 k} \cdot(1.0001)^{k / 2}}{3(2 \pi)^{\frac{k+3}{2}}} \Gamma\left(\frac{k+1}{2}\right)\left[\log \left(\frac{k+1}{2}\right)+\gamma+1\right] \int_{0}^{\infty} y^{\frac{k-5}{2}} \mathrm{e}^{-k \pi y} d y .
$$

The integral above is $\frac{1}{(k \pi)^{\frac{k-3}{2}}} \Gamma\left(\frac{k-3}{2}\right)$, and so the bound on this term is

$$
\frac{14 \cdot 2^{2 k} \cdot(1.0001)^{k / 2}}{3(2 \pi)^{\frac{k+3}{2}}(k \pi)^{\frac{k-3}{2}}} \Gamma\left(\frac{k+1}{2}\right) \Gamma\left(\frac{k-3}{2}\right)\left[\log \left(\frac{k+1}{2}\right)+\gamma+1\right]
$$

and is valid for $k \geq 7$.

After dividing each term above by $\frac{(k-2) !}{(4 \pi)^{k}}$, the main term is increasing linearly (it is about $16 k$ ), and each other term decreases exponentially. The most troublesome term is the term from the third range of values of $n$ from the cusp at infinity, and (after dividing by $\frac{(k-2) !}{(4 \pi)^{k}}$ ) is asymptotic to $c_{1} c_{2}^{k} k^{1 / 4} \ln (k)^{3 / 2}$, where $c_{2} \approx 0.918$, but $c_{1} \approx 1.69 \times 10^{543}$. This term is smaller than the main term only when $k \geq 14,000$.

For this reason, we must explicitly calculate our bounds for $k<14,000$. In this range, we refine our estimate of the troublesome term by using the exact values of the incomplete $\Gamma$-function $\int_{4 \pi n}^{\infty} u^{k-2} \mathrm{e}^{-u} d u$. Also, for $k \leq 2550$, we compute the values of $r_{2 k}(n)$ explicitly for $2 \leq n \leq 2500$ and use these in our bounds. For $k \geq 2552$, we use part 2 of Lemma 3.1 .

Finally, for $k \leq 194$, our numerical bounds are not sufficient and we use Magma to explicitly compute the decomposition of $\theta^{2 k}$ as in equation (1.2) and find that the constants $c_{i}$ are non-negative.

Proof of Theorem 1.1. First, assume that $n$ is odd. Considering the coefficient of $q$ on both sides of (1.2), we obtain

$$
r_{2 k}(1)=4 k=\frac{2 k(-1)^{k / 2}}{\left(2^{k}-1\right) B_{k}}+\sum_{i} c_{i}
$$

By Theorem 1.2, we have

$$
\sum_{i}\left|c_{i}\right|=\sum_{i} c_{i}=4 k-\frac{2 k(-1)^{k / 2}}{\left(2^{k}-1\right) B_{k}} .
$$

Deligne's bound on the $n$th coefficient of $g_{i}(z)$ is bounded by $d(n) n^{\frac{k-1}{2}}$. Plugging this bound into the decomposition and using the fact that the coefficients of $q^{n}$ in $g_{i}(2 z)$ and $g_{i}(4 z)$ are zero if $n$ is odd gives the desired bound on the cusp form contribution to $\theta^{2 k}(z)$. 
Now, suppose that $k / 2$ is odd and $n$ is even. Then $k \equiv 2(\bmod 4)$. We represent the decomposition of the cusp form part of $\theta^{2 k}(z)$ as

$$
C(z)=\sum_{i} r_{i}\left(f_{i}(z)-2^{k} f_{i}(z) \mid V(4)\right)+\sum_{i} s_{i}\left(g_{i}(z)-2^{\frac{k}{2}} \lambda_{i} g_{i}(z) \mid V(2)\right)+\sum_{i} t_{i} h_{i}(z) .
$$

Here, the $f_{i}(z), g_{i}(z)$ and $h_{i}(z)$ are the newforms of levels 1,2 and 4, respectively, and $\lambda_{i}$ is the Atkin-Lehner eigenvalue of $g_{i}(z)$. One can see that the $n$th coefficients of $f_{i}(z)-2^{k} f_{i}(z) \mid V(4)$ and $g_{i}(z)-2^{\frac{k}{2}} \lambda_{i} g_{i}(z) \mid V(2)$ are bounded by $3 d(n) n^{\frac{k-1}{2}}$. Thus, for even $n$, we obtain the bound

$$
\left(\sum_{i} 3 r_{i}+3 s_{i}\right) d(n) n^{\frac{k-1}{2}}
$$

We will show that $\sum_{i} 3 r_{i}+3 s_{i}<4 k-\frac{2 k}{\left(2^{k}-1\right) B_{k}}$.

To compute the constant $\sum_{i} 3 r_{i}+3 s_{i}$, we will compute the trace of $C(z)$ to $S_{k}\left(\Gamma_{0}(2)\right)$, given by $\operatorname{Tr}(C):=C(z)+C(z) \mid\left[\begin{array}{ll}1 & 0 \\ 2 & 1\end{array}\right]$. Straightforward, but somewhat lengthy computations show that

$$
\begin{aligned}
\operatorname{Tr}\left(f_{i}(z)-2^{k} f_{i}(z) \mid V(4)\right) & =3 f_{i}(z)-2 a_{i}(2) f_{i}(z) \mid V(2), \\
\operatorname{Tr}\left(g_{i}(z)-2^{\frac{k}{2}} \lambda_{i} g_{i}(z) \mid V(2)\right. & =3 g_{i}(z), \\
\operatorname{Tr}\left(h_{i}(z)\right) & =0 .
\end{aligned}
$$

It follows from these formulae that $\sum_{i} 3 r_{i}+3 s_{i}$ is the coefficient of $q$ in $\operatorname{Tr}(C)$. We have that

$$
\begin{aligned}
C= & \theta^{2 k}+\frac{1}{2^{k}-1} E_{k}(z)-\frac{2^{k}}{2^{k}-1} E_{k}(4 z), \\
\operatorname{Tr}(C)= & \operatorname{Tr}\left(\theta^{2 k}\right)-\frac{(-1)^{k / 2}}{2^{k}-1} \operatorname{Tr}\left(E_{k}(z)\right)-\frac{2^{k}}{2^{k}-1} \operatorname{Tr}\left(E_{k}(4 z)\right), \\
= & \left(\theta^{2 k}+4^{k} \frac{\eta^{4 k}(4 z)}{\eta^{2 k}(2 z)}\right)+\frac{2}{2^{k}-1} E_{k}(z) \\
& -\frac{2^{k}}{2^{k}-1}\left(\left(1+2^{1-k}\right) E_{k}(z) \mid V(2)-2^{-k} E_{k}(z)\right) .
\end{aligned}
$$

Taking the coefficient of $q$ on both sides of the preceding equation gives

$$
\sum_{i} 3 r_{i}+3 s_{i}=4 k-\frac{6 k}{\left(2^{k}-1\right) B_{k}}<4 k-\frac{2 k}{\left(2^{k}-1\right) B_{k}}
$$

since $k \equiv 2(\bmod 4)$ and hence $B_{k}>0$. This proves Theorem 1.1 in the case that $k \equiv 2(\bmod 4)$ and $n$ is even.

\section{Final remarks}

It is natural to consider if Theorem 1.1 is true in other cases. When $k \equiv 0(\bmod 4)$ and $n$ is even, the main issue is that if $g_{i}$ is a level 1 eigenform and

$$
\tilde{g}_{i}=g_{i}-\frac{4}{3} a(2) g_{i}\left|V(2)+2^{k} g_{i}\right| V(4)=\sum_{n=1}^{\infty} c(n) q^{n},
$$


then the best possible bound on the Fourier coefficients of $\tilde{g}_{i}$ is $|c(n)| \leq 2 d(n) n^{\frac{k-1}{2}}$. In order for this bound to come close to being achieved, it is necessary for $|a(2)|$, the absolute value of the second coefficient in $g_{i}$, to be close to $2^{\frac{k+1}{2}}$. Serre proved in 1997 (see [22]) that if $p$ is a fixed prime, the $p$ th coefficients of newforms become equidistributed (along any sequence of weights and levels whose sum tends to infinity, where the levels are not multiples of $p$ ). It follows from this that there will be level 1 eigenforms with $|a(2)|$ arbitrarily close to $2^{\frac{k+1}{2}}$, but also that there will be few such forms. One approach to extending Theorem 1.1 to the case when $k \equiv 0(\bmod 4)$ is to use the equidistribution of the numbers $|a(2)|$.

It is also natural to consider the problem of deriving a sharp bound in the case that $k$ is odd. In the case when $k$ is even, the contribution from the cusp at zero is (up to a fairly small error) the same as the contribution at the cusp at infinity, since both $\theta^{2 k}$ and the newforms are eigenforms of the Atkin-Lehner involution $W_{4}$. However, when $k$ is odd, the newforms are not eigenforms of $W_{4}$ any longer. This means that the contribution of the cusp at zero is (up to some small error) the contribution of the cusp at infinity times some complex number $\lambda$ of absolute value 1 . This complex number is related to the coefficient of $q^{4}$ of the relevant eigenform $g_{i}$. A similar result could be proven provided one could rule out the possibility that $\lambda$ is close to -1 . In fact, the analogue of Theorem 1.2 is false for $k=17$, although this seems to be a consequence of the smallness of the weight, rather than a value of $\lambda$ too close to -1 .

For half-integral values of $k$ (corresponding to representations of $n$ as the sum of an odd number of squares), the question is still interesting. In this case, the coefficients of the cusp forms involve square roots of central critical $L$-values of quadratic twists of forms of level 1 and level 2. The analogue of Deligne's theorem in this case would be optimal subconvexity bounds on these $L$-values, currently attainable only under the assumption of the generalized Riemann hypothesis.

\section{Acknowledgments}

The author used Magma [23] version 2.17 for computations. The author was supported by NSF grant DMS-0901090.

\section{References}

[1] G. H. Hardy, Ramanujan. Twelve lectures on subjects suggested by his life and work, Cambridge University Press, Cambridge, England, 1940.

[2] J. W. L. Glaisher, On the numbers of representations of a number as a sum of $2 r$ squares, where $2 r$ does not exceed eighteen, Proc. Lond. Math. Soc. 5 (1907), 479-490.

[3] R. A. Rankin, On the representation of a number as the sum of any number of squares, and in particular of twenty, Acta Arith. 7 (1961/1962), 399-407.

[4] G. H. Hardy, On the representation of a number as the sum of any number of squares, and in particular of five, Trans. Amer. Math. Soc. 21(3) (1920), 255-284.

[5] L. J. Mordell, On the representations of numbers as sums of $2 r$ squares, Quart. J. Math. 48 (1917), 93-104.

[6] P. Deligne, La conjecture de Weil. I, Inst. Hautes Études Sci. Publ. Math. 43 (1974), 273-307.

[7] R. A. Rankin, Sums of squares and cusp forms, Amer. J. Math. 87 (1965), 857-860.

[8] S. C. Milne, Infinite families of exact sums of squares formulas, Jacobi elliptic functions, continued fractions, and Schur functions, Ramanujan J. 6(1) (2002), 7-149.

[9] J. Rouse, Bounds for the coefficients of powers of the $\Delta$-function, Bull. Lond. Math. Soc. 40(6) (2008), 1081-1090. 
[10] B. Kim and J. Rouse, Explicit bounds for p-core partitions, accepted in Trans. Amer. Math. Soc., 2009, Preprint.

[11] P. Jenkins and J. Rouse, Bounds for coefficients of cusp forms and extremal lattices, Bull. Lond. Math. Soc. 43(5) (2011), 927-938.

[12] M. Bhargava and J. Hanke, Universal quadratic forms and the 290-Theorem, Preprint.

[13] T. Barnet-Lamb, D. Geraghty, M. Harris and R. Taylor, A family of Calabi-Yau varieties and potential automorphy II, Publ. Res. Inst. Math. Sci. 47(1) (2011), 29-98.

[14] H. Iwaniec, Topics in classical automorphic forms, graduate studies in mathematics, Vol. 17, American Mathematical Society, Providence, RI, 1997.

[15] B. Conrad, Modular forms and the Ramanujan conjecture, Cambridge University Press. In press.

[16] A. O. L. Atkin and J. Lehner, Hecke operators on $\Gamma_{0}(m)$, Math. Ann. 185 (1970), 134-160.

[17] F. Diamond and J. Shurman, A first course in modular forms, Graduate Texts in Mathematics, Vol. 228, Springer-Verlag, New York, 2005.

[18] N. Koblitz, Introduction to elliptic curves and modular forms, Graduate Texts in Mathematics, Vol. 97, Springer-Verlag, New York, 2nd ed., 1993.

[19] M. Abramowitz and I. A. Stegun, Handbook of mathematical functions with formulas, graphs, and mathematical tables, National Bureau of Standards Applied Mathematics Series, Vol. 55, For sale by the Superintendent of Documents, U.S. Government Printing Office, Washington, D.C., 1964.

[20] R. A. Rankin, Contributions to the theory of Ramanujan's function $\tau(n)$ and similar arithmetical functions. I. The zeros of the function $\sum_{n=1}^{\infty} \tau(n) / n^{s}$ on the line $\operatorname{Re}(s)=13 / 2$. II. The order of the Fourier coefficients of integral modular forms, Proc. Cambridge Phil. Soc. 35 (1939), 351-372.

[21] W. C. W. Li, Newforms and functional equations, Math. Ann. 212 (1975), 285-315.

[22] J.-P. Serre, Répartition asymptotique des valeurs propres de l'opérateur de Hecke $T_{p}$, J. Amer. Math. Soc. 10(1) (1997), 75-102.

[23] W. Bosma, J. Cannon and C. Playoust, The Magma algebra system. I. The user language, J. Symbolic Comput. 24(3-4) (1997), 235-265. Computational algebra and number theory (London, 1993).

Department of Mathematics, Wake Forest University, Winston-Salem, NC 27109, USA E-mail address: rouseja@wfu.edu 